\title{
GENERAL PHYSICAL LAYOUT:
}

- The backplane is comprised of 2 identical sections, the upper half designated $\mathrm{J} 1$, and the lower half designated $j 3$.

- For each section, there are a total of 16 slots, designated 1 through 16 , from left to right.

- Odd numbered slots are left handed (Connectors to the left of the card). These slots utilize inverse DIN connectors.

- Even numbered slots are right handed (Connectors to the right of the card). These slots utilize standard DIN connectors.

- There are 260 pin daisy-chain headers associated with each section, 3M part number 3597-6003.

- The headers associated with the J2 (upper) section are designated J2CHAINR and J2CHAINL.

- The headers associated with the J3 (lower) section are designated J3CHAINL and J3CHAINR.

- Mechanical layout presented in drawing number 3823.113-MD-330045.

\section{TRIGGER SIGNALS:}

- There are 21 trigger signal which originate from a given slot and are routed to the slot immediately to the left and 21 trigger signals which originate from a given slot and are routed to the slot immediately to the right.

- There are 21 trigger signals which originate from the slot immediately to the left of a given slot and 21 trigger signals which originate from the slot immediately to the right a given slot.

- The signal are designated TRIGjk-n, when $\mathrm{j}$ is the source slot, $\mathrm{k}$ is the destination slot, and $\mathrm{n}$ is the signal number.

- These signals shall be implemented as $100 \mathrm{ohm}$ microstip above ground plane.

- No terminations.

\section{POWER:}

- Each slot is supplied with DC power on the connector pins designated as VCC and GND.

- VCC and GND originates at rear of the backplane via press-in power terminals. Their locations are shown in drawing number 3823.113-MD-330045.

- Power shall be bypassed at 2 locations with a $10 \mathrm{uF}$ tantalum capacitor in parallel with a $.01 \mathrm{uF} 100 \mathrm{~V}$ ceramic capacitor.

- The total ampacity supplied by each DIN connector is 3.6 amps. 


\section{CONNECTOR CONFIGURATION:}
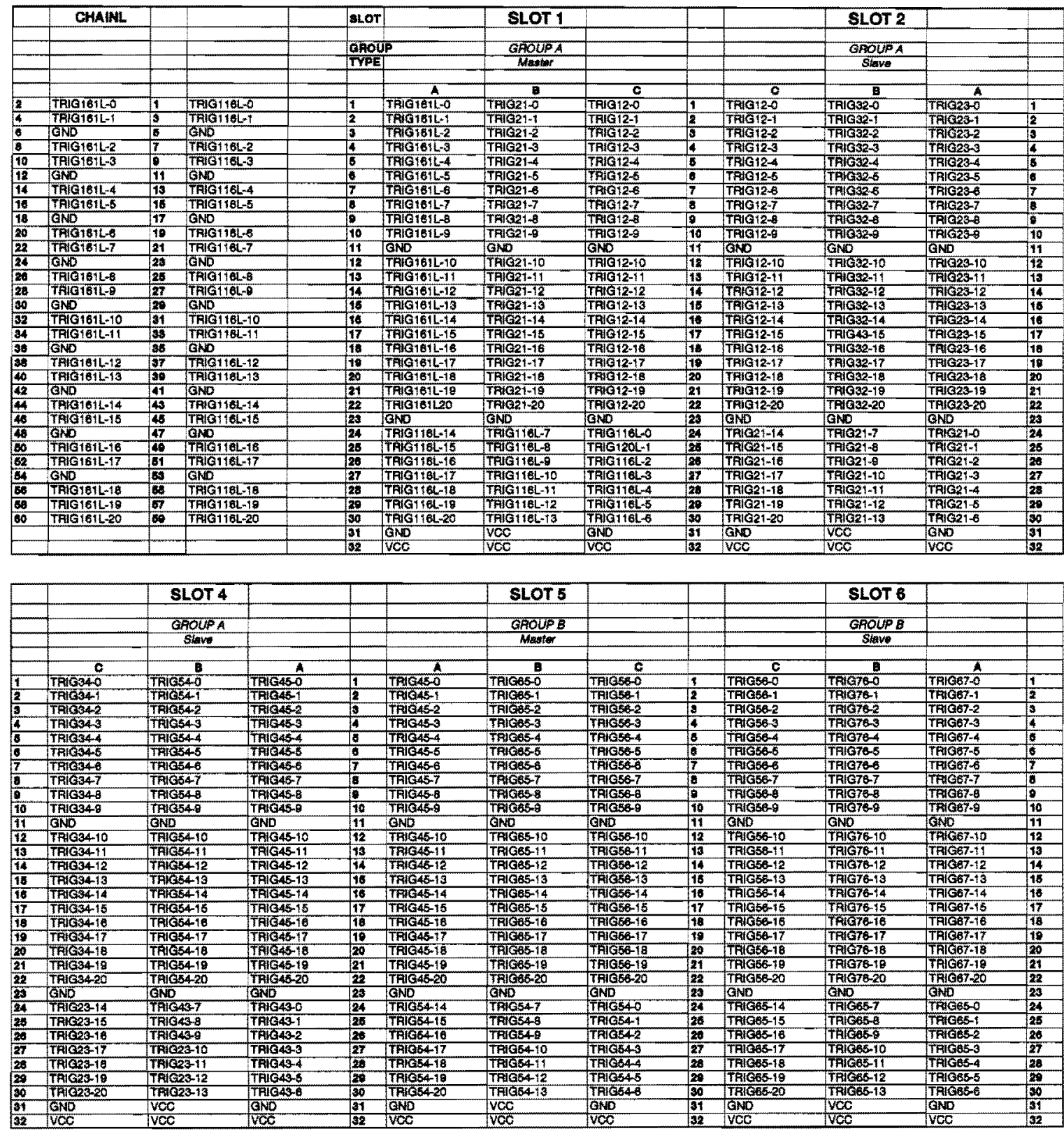


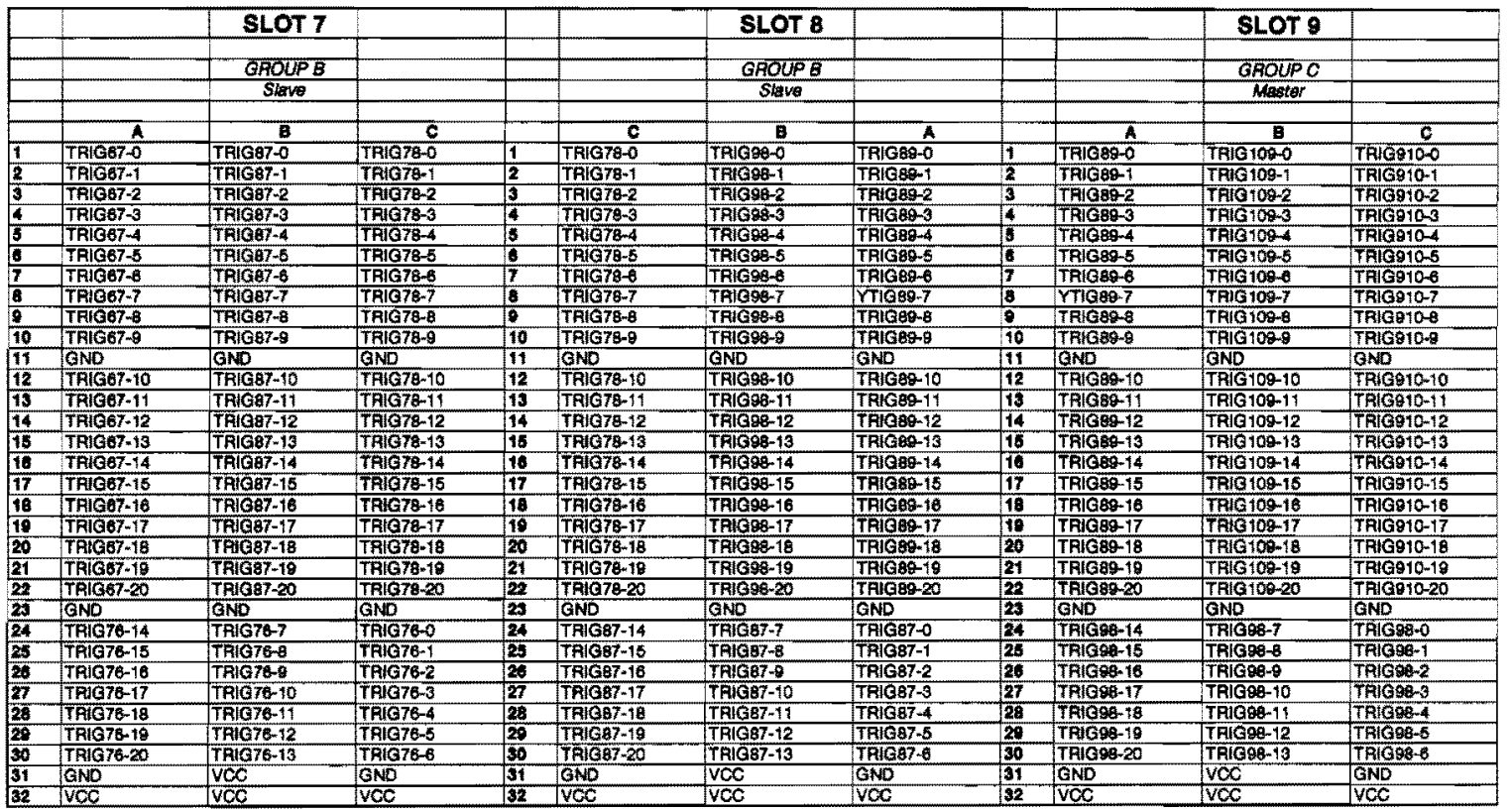

\begin{tabular}{|c|c|c|c|c|c|c|c|c|c|c|c|}
\hline & & SLOT 10 & & & & SLOT 11 & & & & SLOT 12 & \\
\hline & & GHOUPC & & & & GROUPC & & & & GROUPC & \\
\hline & & Slave & & & & Siave & & & & Slave & \\
\hline & & & & & & & & & & & \\
\hline & C & $\begin{array}{r} \\
\end{array}$ & $\begin{array}{l}\mathbf{A} \\
\end{array}$ & & $\mathrm{A}$ & $B$ & $c$ & & $\mathrm{C}$ & 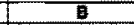 & $A$ \\
\hline 1 & TRIG910-0 & TRIGIt100 & TAIG10:1-0 & 1 & TRIG10110 & TRIGIR/10 & TRIG1112-0 & 11 & TAIGII12-O & TAIG13120 & TFiG1213-0 \\
\hline 2 & IPIG910-1 & TRIG1110-1 & TRIG1011-1 & 12 & ThiG1011-1 & TRIG1211-1 & TFIG1+12-1 & $\frac{1}{2}$ & TRIGI112-1 & TAIGIBI2-1 & TRIG:112-1 \\
\hline 3 & TRIO910-2 & TRIGITIO-2 & TAIG1011-2 & 3 & Thiolo11-2 & TAIG1211-2 & TPIG:112-2 & 5 & TRIGI1122-2 & THIG13:2.2 & TFiGIt12-2 \\
\hline 4 & TPAOOP103 & TRIGI'10.3 & TRIQ1011-3 & 4 & Talo1011-3 & TPHS1219-3 & IFIGI112-3 & 4 & TRIG1112-8 & TPIGIOT23 & TAIGI112-3 \\
\hline 5 & TAIGS10-4 & TRPG1110-3 & TAHG1011-4 & 6 & TäG1011-4 & TPIG121T-4 & TRIGII12-4 & 8 & TrRGI112-4 & TRIG1312-4 & TRIG1112-4 \\
\hline 6 & TRाGQ10-6 & TRIG1110-6 & TFIG1O11-5 & If & TPIGToti-5 & TRIG1211-5 & TRIG1T112-5 & 6 & TREG & TRGG1312-5 & TRIG1112-E \\
\hline 7 & TRIOSID-6 & TRIQ1110-6 & TRQO1011-6 6 & 7 & TRIG1011-6 & Tमाब1211-6 & TRIGI112-6 & 7 & TPGGIII26 & Trato1312-6 & TRIG1112-6 \\
\hline 8 & Thígsta-7 & TRIG1110-7 & TRIO1011-7 & 16 & Tन161011-7 & TRHO1219-7 & Rाa1112-7 & 8 & TFIG1112.7 & TRIG $1312-7$ & TFAGIII12-7 \\
\hline 5 & TRESTOS & TAIGIITO- & ThIGIOT-8 & 9 & TAIGTOH:B & TPIO1211-8 & TPIGI112-8 & e & TAIG1112-8 & ITIG $1312-8$ & TPIGIIIR-8 \\
\hline 10 & TRIGS10-9 & TFIG1110-9 & FIGIOII-9 & 10 & TRG1011-9 & TPIG1214+9 & TPRGII12-9 & 10 & TrAGH112-9 & TAlG1312-9 & TPIOIIIR-S \\
\hline$\frac{2}{11}$ & SND & GNO & GND & $\frac{19}{19}$ & GND & OND & GNO & 11 & GND & GNO & GND \\
\hline 12 & ITRG910-10 & TAIGM110-10 & ThAO1071-10 & 12 & TRIG10:1-10 & TRIG 12) Y-10 & TRIG1Y12-10 & 12 & TRIG1112*10 & TRIOL1312-10 & TRIa11/2:10 \\
\hline 13 & TAIGP10-11 & Trionito-11 & TFig101t-11 & $1+3$ & ThIS1014-11 & TRIGI21-11 & TRIO1112-11 & 13 & TAIGI112-11 & TRIG1312-11 & TAIG1112-1: \\
\hline 14 & TAIGo10-12 & TRIG1110-12 & TPIG10I1-12 & 114 & TथाG1011-12 & TRIG1211-12 & TAIG111k-12 & 14 & TRIG $1112-12$ & TRIO1312-12 & TRIG $1112-12$ \\
\hline 15 & ThIG910-13 & TRIG1110-13 & TFiG 101t-13 & 15 & TAIGIOI $1 \mathrm{~m}$ & TRIGI211-13 & TRIGIIIZ-15 & 115 & TPIG1112-13 & TRIG1312-13 & TRIG:112-13 \\
\hline 18 & TRIG910-14 & TRIG1110-14 & TAIG $1011+14$ & 116 & TRiG1011-14 & TRIGIZI1-14 & TRIG1112 14 & 16 & ITRIGI112-14 & ITRG1312-14 & ITAG:t12-14 \\
\hline 17 & ThiG910-15 & TRIG1110-15 & TAIG1O11-15 & 117 & TRGG1011-15 & TRIGLIIIIIS & TPIGI112-16 & 17 & TaIG1112-15 & TRIGIS12-15 & TFIGIT12-15 \\
\hline 18 & TPIG910-18 & TRIG:110-16 & TRIG1011-1B & 18 & TRIG1011-18 & TRIG1211-18 & TRAG1112 16 & 18 & TRIGII12-18 & TRIGIS12.18 & TRIG $1112-18$ \\
\hline 10 & TRIG910-17 & TRIG1110-17 & TRIG101 $1-17$ & 10 & IRIG1011-17 & 7RGG1211-17 & TFIG1112-17 & 18 & ThIG1112-17 & TRIGI3I2 17 & TRIGI112-17 \\
\hline 20 & TRIGQ10.18 & TRAG1110-18 & TATG9011-1B & 20 & TROAO11-18 & TRIG121 1-18 & TRIG1112-18 & 10 & TPIGIII218 & TRIG1312-18 & TFiGit12-18 \\
\hline 21 & TriGgro-19 & TRAQ1110-18 & Trigro11-19 & 21 & TRAC61011-19 & ThIG1211-19 & TRIG1112-18 & 21 & FFG1112-10 & TRIG1312-19 & TRIG1112-19 \\
\hline 22 & Trios 10-20 & TRAG1910-20 & TrLG101:-20 & 122 & TalG1011+20 & TRIGI $121+20$ & TRIG1112-20 & 22 & TRIGIII2-20 & TRIG $1312-20$ & TPIGIt12-20 \\
\hline 23 & GNO & GND & GNO & 20 & GNO & GND & GND & 23 & GND & GND & GND \\
\hline 24 & TFIG $100-14$ & TRIG100-7 & TRIG10000 & 26 & TAlGt110-14 & TAIG1110-7 & TRIG1110-0 & 24 & TFIG 1211-14 & TPHG1211.7 7 & TRGIZ1100 \\
\hline 23 & TREGTo-15 & TRIG100-8 & TR1O109-1 & 25 & TAIGI1L-15 & TRtQG1110-8 & rriatilat & 25 & TARG $1211-15$ & ITIG1211-8 & TPlai211-1 \\
\hline 28 & TRIG $100-16$ & TRIC1000 & ThIO100.2 & 20 & TRIGI110-16 & TRAOT110-8 & FRIGIIta2 & 20 & TAIS12111\%8 & TrROL1211+0 & TRG \\
\hline$\frac{17}{E 7}$ & TRIOL100-17 & Tि1ब100-10 & TRIGIOQ-3 & 27 & TRIG111017 & TRIOIIIO-10 & Fról1103 & 2 & TRIG1211-17 & TriGiz1:-10 & TRIG1211-3 \\
\hline 28 & ITFG 10218 & TRIG too-11 & TRIGIOS-4 & $\frac{18}{28}$ & TRIGIIL-18 & TRIG1t+o-11 & FROS1110-3 & 20 & TAIG1211-18 & TRIC1211411 & TRIOIZ11-4 \\
\hline 20 & FFig100-10 & ThIGroo-12 & TRIBIOAO & 20 & Thilllo-19 & TAIG:110-12 & TPIOIIIO-5 & 23 & TRIG12T1-19 & TFIOL1: +12 & TRIG1211m5 \\
\hline 30 & TPAG100-20 & TAIG $109-13$ & TRIG109-8 & 50 & Thiol110.20 & ThIGHIL-13 & TAGG1110- & 130 & TRIG1211-20 & TRIG1211/13 & TRIG1211-8 \\
\hline 31 & CND & Voc & OND & S1 & GNO & VCC & GNO & Bi & GND & VCC & GND \\
\hline 32 & IVCo & Voc & VCC & $\sqrt{32}$ & $\mathrm{VCC}$ & vec & $\mathrm{VCC}$ & 35 & $V C C$ & VCo & Vec \\
\hline
\end{tabular}




\begin{tabular}{|c|c|c|c|c|c|c|c|c|c|c|c|}
\hline & & SLOT 13 & & & & SLOT 14 & & & & SLOT 15 & \\
\hline & & GROUPD & & & & GROUPD & & & & GROUPD & \\
\hline & & MAsies & & & & Siate & & & & Since o & \\
\hline & & & & & & & & & & & \\
\hline & $\mathbf{A}$ & 8 & $c$ & & $c$ & E & $\begin{array}{l}\mathbf{A} \\
\end{array}$ & & $\bar{A}$ & G & $c$ \\
\hline 1 & TRIG121300 & TAIG1413-0 & TRIG13140 & 11 & TRIGIX14-0 & TRIG16140 & TRGG14150 & 5 & TRIG141800 & TAIG1610-0 & TRIGISIG-0 \\
\hline 2 & TRIG1112-1 & Tria 1413-1 & TAIGIOI41 & 2 & TAIG1314-1 & ThIG1514-1 & TRIG14IS! & 2 & TAIG1416-1 & TRIG1A15: & TRIG1516+ \\
\hline 3 & FPle1112.2 & Thla141322 & TRIG10142 & 3 & TRIE1314-2 & TRLG1544-2 & TRIG 1415-2 & 3 & TAIGI415-2 & TRiG1615-2 & TALGiEIG-2 \\
\hline 4 & Th10m112-3 & TAIG 1413.3 & TRIG1314-3 & 4 & TRIG1314-3 & TAla $1514-3$ & TRIG1415-3 & 4 & TAIG1416-3 & IRIG1615-3 & TRIG1516.3 \\
\hline 5 & TRIGIII2-4 & TRIOT:143.4 & TRIO1314-4 & s & TRIGI3144 & TRIQI514-4 & TRIG1415-4 & 5 & Thisi415-4 & TAIO1015-4 & TRIG1510-4 \\
\hline 8 & TAIG1112-5 & TRIOTA13-5 & TRIG13145 & 16 & TFIG13:45 & TRIQ15145 & TAIG1415-5 & 6 & TAIO1416-5 & TRIG1615-5 & TAIGIO16-5 \\
\hline 7 & TRMG1112-6 & TRIG1413-8 & TRIG1S14-6 & 7 & TRIGIS14- & TRIG1514-8 & TRIG:416- & 7 & TRIG1415.5 & TRIG $1615-6$ & TRIG $1010-8$ \\
\hline 8 & TRIOI1112.7 & TAIG 1413-7 & TAlG13:4.7 & 8 & TAIG1314-7 & ThIG 15147 & TA1G1415-? & is & TRE 1416.7 & Thict615.7 & TRIG1516-7 \\
\hline$\theta$ & TRIGII12-8 & TRIGt413-8 & TFlब1314-8 & 9 & TRIG13I4-8 & TFiGt514-8 & TRIG1A15-8 & 8 & TRIGILIG-8 & TRIG1815-8 & TRIG1616-8 \\
\hline 10 & TRIG1112-9 & TRIG 1413: & TRlG1314-8 & 10 & TAIG 13149 & TRIG1514-8 & TRIG 1415-9 & 10 & TRIG14IE-9 & TRIS 1015-8 & TAIG161e-9 \\
\hline II & CNO & GND & GNO & 11 & GND & GND & GND & 11 & GNO & GNO & GND \\
\hline 12 & TRIGI112-10 & TRIG1413-10 & TRIG13:4410 & 112 & TRIG1314-10 & TRIG1514-10 & TRIG1416-10 & 12 & TRIG1416-10 & TRIGIB1E-10 & TRIG $1516-10$ \\
\hline 13 & TRIG1112-11 & TAlG1413-11 & TAIG1314-11 & 13 & TRIG $1314-11$ & TRIG1514-11 & TRIG 1415-11 & 13 & TRIG14tG.11 & TRIG:616-11 & TRIG1516-11 \\
\hline 11 & TRIOUIIZ12 & Tि161413-12 & TRIG1314-12 & 14 & Tค161314-12 & TA1G1514-12 & TRIG IA16-12 & 116 & TAIG IAIS-12 & TRIOA 1616-12 & TRIS1516-12 \\
\hline 16 & TRIG1112-13 & TRIG141013 & THIG1314-13 & 16 & TRIG1314/13 & TRIG151413 & TRIGIS1E-13 & 16 & TPG 1410-13 & TRIG1S1E-13 & TPIO1616-13 \\
\hline 16 & Triat112-14 & TRIG1413-14 & TAIG1314-16 & 10 & TAIS 13ta-14 & TRIO1514-14 & TAlG 14 is-14 & 18 & TRla1415-14 & TRIG1815-14 & Ta|G $1516-14$ \\
\hline 17 & TAIG1112 15 & TRIOL1413-15 & TAIG1314-15 & 17 & TAIG & ThIG $1514+15$ & TRIG1415-15 & 17 & TRIOI 1415-15 & TAIG1B:S-15 & TRIG1S18 15 \\
\hline 16 & TAlG1112-10 & TRIG1413-18 & Trio1314-16 & 10 & TRIG1314-18 & TAIG1614-16 & TRlG1415-16 & 18 & TAIG $1416-18$ & TR101818-16 & TRIGI618-16 \\
\hline 10 & TAIG1112-17 & TFilO1413-17 & TF161314-17 & 10 & TRIG $1314-17$ & TRIGIS14-17 & TRIG1416-17 & 110 & TRIG1415-17 & TAIG1815-17 & TRIG $1518-17$ \\
\hline 20 & TRIG1112-18 & TคIG1413-18 & TRIG1314-18 & 20 & TR161314-18 & TRIG1S14-18 & TRIGY41E-18 & 20 & TRIG1416-18 & TAIG161E-18 & TFIG1516-18 \\
\hline 21 & TALG1112-19 & TRGG1413-18 & TAIG131419 & 24 & TRIG1314-19 & TRIG1514-19 & TAIG1415-19 & 21 & TRIG1415-19 & TFIG1616-19 & THIG1518.te \\
\hline 20 & TAIG1112-20 & TRIO141320 & TR6 $1914-20$ & 122 & TีIG $1314-20$ & TRIG1514-20 & TRIG 1415-20 & 22 & TRIOI1415-20 & TAIOT616:20 & Tค1G1518-20 \\
\hline 23 & QND & GND & GND & 23 & GND & GNO & GND & 23 & GND & GNO & GND \\
\hline 24 & TA1G1312-14 & TRIG1312-7 & TAIG 13120 & 24 & Tri61413-16 & TFIG1613? & TRIG 1413-0 & 24 & TFIG $514-14$ & FFIG15147 & TAIG 15140 \\
\hline 25 & TF161312m 15 & TFIO1312-8 & TALG1312-1 & 25 & TRIC1413-15 & TFIO14138 & TRIG 1413- & 23 & TRIO1S:4-15 & ThIO1514-8 & TALO1514-1 \\
\hline $\mathrm{tat}$ & TAGG1312-16 & TAIG $1312-8$ & TRIG1312-2 & 120 & TAIGIA13-16 & TRIG1413-9 & TRlG 1413-2 & 28 & TAIG15:4-18 & TFIGIS14-2 & TRIG1514-2 \\
\hline 27 & TRIG $1312+17$ & Thlaks12-10 & TAIC1312-3 & 27 & TAlO1413-17 & TRIG1A13-10 & TRIG 14133 & 27 & TRIG 1514-17 & TRIO1514-10 & TFIOIS14-3 \\
\hline 28 & TRIG 13:2-18 & TRIG1312-1 & TA1G1312-4 & 28 & TAIG1413:18 & TRIGTAL-11 & TRlo 1413-4 & 28 & TAIG +514-18 & THIO1514-11 & TAIG1514- \\
\hline 29 & THGGIS12-18 & TALY1312-12 & TAIGI312-5 & 20 & TRIG $1413-18$ & TPIG1413-12 & TRIG1413-6 & 20 & TAIG1614-18 & TRIG:B14-12 & TALG $1514-5$ \\
\hline 30 & TRIGI312-20 & Thl61912-13 & TRIG1312-5 & 130 & Thil $14+3-20$ & TRIG1413-13 & TRIG1413-6 & 30 & TRIG151420 & TRIG1ST4-13 & TRlo1514-6 \\
\hline 31 & GND & VCo & GND & $\sqrt{34}$ & GND & Voo & GND & 31 & GNO & VOC & GND \\
\hline 32 & VOC & $\mathrm{VCC}$ & $\mathrm{V} C$ & $\sqrt{32}$ & VCC & VCC & $\sqrt{\mathrm{CCC}}$ & 132 & VCC & $V C C$ & vac \\
\hline
\end{tabular}

\begin{tabular}{|c|c|c|c|c|c|c|c|}
\hline & & SLOT 16 & & & CHAWNA & & \\
\hline & & & & & & & \\
\hline & & GROUPD & & & & & \\
\hline & & Slave & & & & & \\
\hline & & & & & & & \\
\hline & $c$ & $B$ & $A$ & & & & \\
\hline 1 & TAIG15180 & TRIG17:B-0 & TRIGIBI7-0 & 2 & TRIGIBIR-O & 1 & TRIG118A-0 \\
\hline 2 & TARG1518-1 & TRIG1716-1 & IRIG1617-1 & 4 & TRIG1GIR-1 & 3 & TRIG116R-1 \\
\hline 3 & TRIGISIB-2 & TAIG1716-2 & Tfla1617-2 & 6 & GNO & $\frac{5}{15}$ & GND \\
\hline 4 & TRIG1516- & TAIGI & TRIQ1B17-3 & B & TAIG181P2 & 7 & TRIG116R2 \\
\hline 5 & TAIGISTB-4 & TRIG $+716-4$ & TRा01617-4 & 10 & TRIGI6IF-3 & 8 & TRIG11QR-3 \\
\hline 6 & ThiG:518-5 & TAIGI716-5 & TPIG1617.5 & 12 & GND & 11 & ciNo \\
\hline 7 & TRाG1516-8 & TAIG1716-6 & TRIG1617.6 & 14 & TRIG161R-4 & 13 & TRIOI ISA-4 \\
\hline 8 & TAIG151B-7 & TAIGI716-7 & TRIGI817.7 & $\sqrt{16}$ & TRIG161R-5 & is & TRIGTIBA-5 \\
\hline 8 & TALG151898 & TFIO1718-8 & TRाG1517-8 & 18 & GND & 17 & GND \\
\hline 10 & TAIOISIG-9 & TalGiziens & TAIG1617-8 & 20 & TAIGIBIRE & 19 & TFIG11BAB \\
\hline 11 & GND & GND & GND & 22 & TRIG161R-? & 21 & TRIO11BR-7 \\
\hline 12 & TRIG1516-10 & TAIG1716-10 & TRIG1B17-10 & 22 & GNO & 23 & GNO \\
\hline 13 & TAIG1516-11 & TAIG1718-11 & TRIG1817.11 & $\sqrt{20}$ & TRIGI6IR-8 & $\sqrt{26}$ & TAIGI1BA-8 \\
\hline 14 & ThIG1516-12 & TAIG $1716-12$ & TRIG $1617-12$ & 28 & TAIGI6IR-9 & 27 & TRIGI 16 A9 \\
\hline 18 & TAIG1516-13 & TRIO1718-13 & TAIG1BI7-19 & 130 & GND & 20 & GNO \\
\hline 10 & ITRIG1516-14 & TAIG171B:14 & TRI01617-14 & 32 & TRIG1B1R-10 & 31 & TRGG11BA-10 \\
\hline 17 & TRIG1516-15 & TAlG17/6-16 & TRIGI6I7-18 & 34 & TRlG161R-11 & 33 & TRIGI $16 \mathrm{AH}: 1$ \\
\hline 18 & TRIG1516-16 & TAIG1716-16 & ThIG1617416 & 36 & GND & 35 & GND \\
\hline 10 & TRIG161E-17 & TAIS1716-17 & IA101617-17 & 38 & TRIG161R-12 & 37 & TAIGI BR-12 \\
\hline 20 & TRIG1616-18 & TAIGI $76-18$ & TRIG1617-18 & 40 & TRIG161R-13 & 30 & THIGI $69-13$ \\
\hline 21 & TRIG $1518-18$ & TAIG1716-10 & TR1G1617+19 & 42 & GND & 11 & GND \\
\hline 22 & TRIO1616-20 & TRIOT716-20 & TAIG1697-20 & 44 & TA1G161R-14 & 49 & TFIOT1बA-14 \\
\hline 23 & GND & GND & CNO & 46 & TRIG161F-IS & 46 & TRIQ118R-15 \\
\hline 24 & TAlG1815-14 & TAIBIBE-? & Th101615-0 & 48 & OND & 47 & GNO \\
\hline 26 & TAIG1615-15 & TRIS1015-8 & TAIGIBIS-1 & 80 & TAIG EIF-16 & 40 & TAIG1 16P-16 \\
\hline 20 & TRIG1615.16 & TFIG16158 & TRIGI616-2 & 32 & TAIG161P-17 & 81 & TAIG118A17 \\
\hline$\frac{17}{27}$ & TAIGIB1G:17 & TALG1815-10 & Tค1G1815-3 & 8 & GND & 63 & GNO \\
\hline 28 & THIGI815-18 & TRIG1615-11 & TAIG:615-4 & 8 & TRIG161R-18 & 5 & TRIGIIBA-18 \\
\hline 2 & TRIG1615-19 & TRIGBBIE-12 & TA1G1615-5 & 68 & TAIGIS1R-18 & 57 & TRGGHAR 19 \\
\hline$\frac{10}{30}$ & TRIG1615-20 & TRLG1616-33 & TRIO1815-6 & $\infty$ & TAIGIBIP-20 & 58 & TRIG116R-20 \\
\hline
\end{tabular}

